\title{
Segmentation, Registration, and Deformation Analysis of 3D MR Images of Mice
}

\author{
Ghassan Hamarneh ${ }^{1}$, Josette Chen ${ }^{1}$, Brian Neiman ${ }^{1}$, \\ Jeff Henderson ${ }^{2}$, and Mark Henkelman ${ }^{1}$ \\ 1 Mouse Imaging Centre, Hospital for Sick Children, Toronto, ON, M5G 1X8, Canada \\ 2 Faculty of Pharmacy, University of Toronto, Toronto, ON, M5S 2S2, Canada
}

\begin{abstract}
We demonstrate our mouse MR image and shape analysis pipeline. The long term goal of our work is the description of structural shape variations in normal, genetically identical mice and the subsequent detection of pathological phenotypes in genetically modified mice. The pipeline begins with the acquisition and reconstruction of high resolution MR images of mice. Regions of the images containing organs under study are enhanced via edge-preserving smoothing and mouse organs are segmented using a deformable mesh model. Images are registered using a multi-resolution approach and displacement fields are used to calculate local volume changes. Detailed descriptions and results for each step of the pipeline are presented.
\end{abstract}

\section{Introduction}

Genetics may hold the key to unlocking the mysteries of many human diseases. Understanding the genetic basis of human anatomy, physiology, and disease may translate into better diagnostic, prognostic, and therapeutic treatments. Genetic makeup of non-human organisms such as fruit flies, yeast, worms, zebrafish and mice provide insights into the origins of human disease. Recent advances in genetic research show that similar genes regulate basic life processes and patterns of growth and development across a variety of species. Mice are the mammalian model of choice for the study of functional genomics for several reasons: Mice genes and pathways are similar to humans[1], mice can exhibit clinical symptoms of many human diseases[2,7,14], the murine genome has recently been completed[13], and a number of genetic tools presently exist which allow the rapid modification of the murine genome and mapping of mutant alleles. Using genome-wide chemical mutagenesis in mice via ENU[12], scientists are developing new murine models which mimic aspects of human disease. Such experiments are already underway at the Mouse Mutagenesis for Developmental Defects at the Baylor College of Medicine in Texas, the Harwell Mutagenesis Programme in the United Kingdom, the ENU-Mouse Mutagenesis Screen in Germany, the Centre for Models of Human Disease at the University of Toronto in Canada and elsewhere. Screening genetically altered mice for clinical disorders through physiological (e.g. cardiovascular, renal, bone) and behavioral (e.g. learning, memory) screens, is complemented by powerful, non-invasive assessment of mouse models 
through the acquisition and analysis of high-resolution medical images such as ultrasound bio-microscopy (UBM), micro-computed tomography $(\mu \mathrm{CT})$, optical projection tomography (OPT), and magnetic resonance (MR) microscopy.

In our current work we apply a number of computational image analysis techniques in an effort to describe structural shape variations in normal, genetically identical mice for the subsequent detection of pathological phenotypes in genetically modified mice. Other efforts taking place worldwide to analyze images of mice for the study of functional genomics include the Mouse Atlas Project at UCLA and the Edinburgh Mouse Atlas Project. Our work is designed to be part of the primary screening stage of randomly ENU-mutagenized mice for the detection of gross pathology. We make primary use of 3D image registration[8], and model-based segmentation techniques[9]. In particular, we demonstrate our MR image and shape analysis pipeline of mice kidneys and brains.

This paper is organized as follows. The parallel MR acquisition and the preprocessing of the the mouse images are discussed in 2.1 and 2.2. Methodologies for segmenting and registering the images are discussed in 2.3 and 2.4. Total volume and local volume change measurements of segmented and registered organs are described in 2.5. Implementation details are presented in 3. Results of the output from each stage of the pipeline are presented throughout the paper in the appropriate sections.

\section{Methods}

\subsection{Magnetic Resonance Imaging of Mice}

Murine MRI images were derived for three anatomic fields: Whole body MR scans $(110 \mu \mathrm{m}$ isotropic resolution, $256 \times 256 \times 1024$ voxels[6], denoted $\mathrm{D}$ in the text), a single multiple-mouse scan experiment conducted to image four mice kidneys in situ $(117 \mu \mathrm{m}$ isotropic, $256 \times 256 \times 384$ voxels, $7-\mathrm{T}$, spin echo, TR/TE 400/17ms, 21.8 hours, denoted M1), and scans of excised brains of wildtype and transgenic knockout $(40 \mu \mathrm{m}$ isotropic, $384 \times 192 \times 192$ voxels, $7-\mathrm{T}$, spin echo, TR/TE 1500/30ms, 15.4 hours, denoted M2). Example images are shown in Figure 1.

\subsection{Image Pre-processing}

To improve signal to noise ratio prior to segmentation and registration, images are smoothed using anisotropic non-linear diffusion filtering. The principle is to smooth out noise locally by diffusive flow while at the same time prevent flow across object boundaries. The image volumes are filtered according to

$$
\partial_{t} I=\operatorname{div}\left(g\left(\left|\nabla\left(G_{\sigma} * I\right)\right|\right) \nabla I\right)
$$

where $I$ is the image volume, $G_{\sigma}$ is a Gaussian kernel of width $\sigma$, and $g$ is a diffusivity function[11]. Edge-preserving smoothing prior to subsequent analysis yielded more robust results. Figure 2 shows different levels of anisotropic smoothing. 


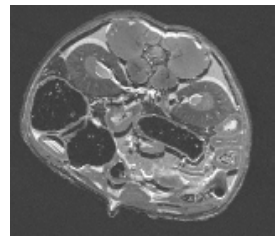

(a)

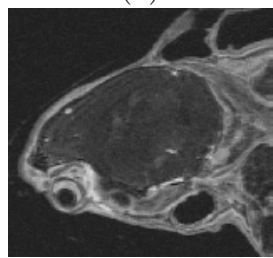

(d)

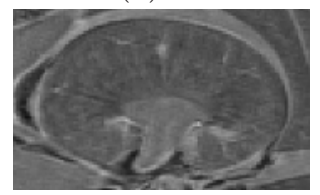

(g)

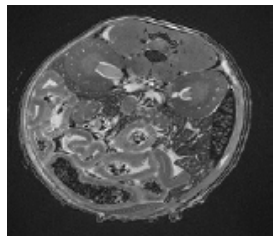

(b)

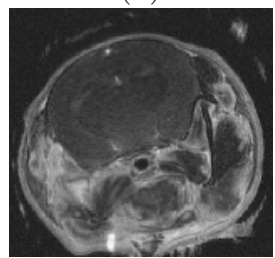

(e)

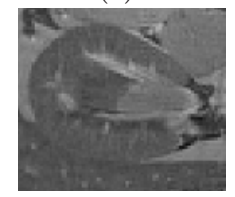

(h)

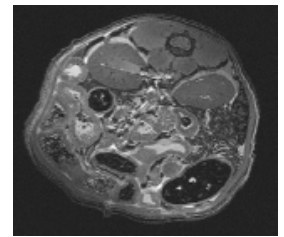

(c)

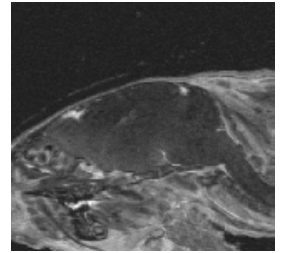

(f)

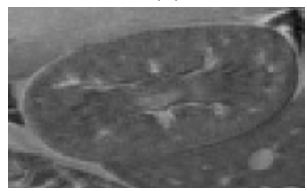

(i)

Fig. 1. MR mice image examples. (a-c) Transversal planes through abdomen, showing kidneys in images acquired in parallel using multiple-mouse imaging system[M1]. (d-f) Coronal, transversal, and sagittal planes through an image of a mouse brain and (g-i) a mouse kidney[D].
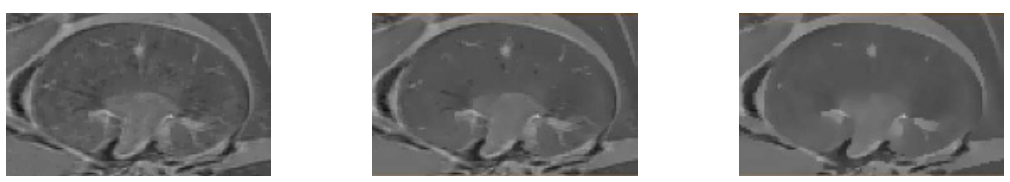

Fig. 2. Diffusion filtering of a mouse kidney (more diffusion from left to right)[D].

\subsection{Deformable Model Segmentation}

Quantifying organ volume and shape variation properties in normal population of mice necessitates the identification of organ boundaries. We use deformable simplex mesh models for segmentation. The underlying geometry of the model is a 2-simplex mesh. A spherical mesh model is initialized manually in the proximity of the target structure in the $3 \mathrm{D}$ image. The mesh is iteratively deformed to latch to the structure's surface while progressively allowing more localized deformations. Two types of local forces are calculated and applied at each vertex $\left(V_{i}\right)$ of the mesh: Internal forces $\left(f_{\text {int }}\right)$ derived from surface smoothness energy terms, and external forces $\left(f_{\text {ext }}\right)$ along surface normals and proportional to the distance from a vertex to high gradient image voxels. Deformable simplex meshes apply 
Newtonian law of motion to update the positions of the mesh vertices[4]:

$$
m \frac{d^{2} V_{i}}{d t^{2}}+\delta \frac{d V_{i}}{d t}=\alpha f_{\text {int }}+\beta f_{\text {ext }}
$$

where $m$ is mass, $\delta$ is a damping coefficient, $\alpha, \beta$ are weights. Global transformations are obtained through the application of global forces $\left(f_{\text {global }}\right)$ resulting in a deformation with lower degrees of freedom. A parameter $\lambda$ sets the relative influence of global and local forces[10]:

$$
V_{i}^{t+1}=V_{i}^{t}+(1-\delta)\left(V_{i}^{t}-V_{i}^{t-1}\right)+\lambda\left(\alpha f_{i}^{i n t}+\beta f_{i}^{e x t}\right)+(1-\lambda) f_{i}^{g l o b a l}
$$

Proper image pre-processing to enhance edges and choice of model parameters were necessary to obtain the desired results. Figure 3 and Figure 4 show examples of kidney and brain segmentations.

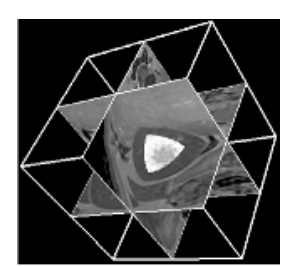

(a)

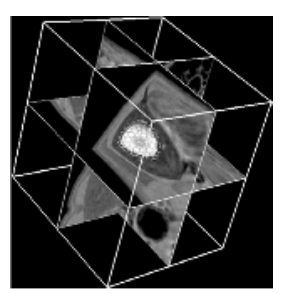

(d)

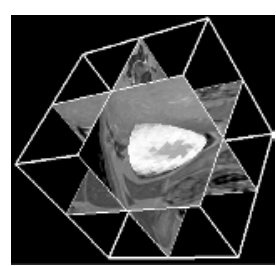

(b)

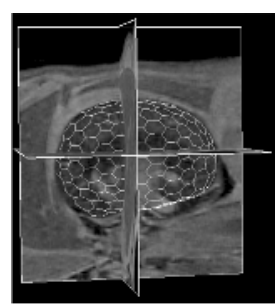

(e)

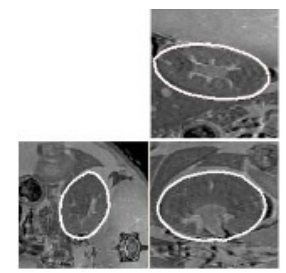

(c)

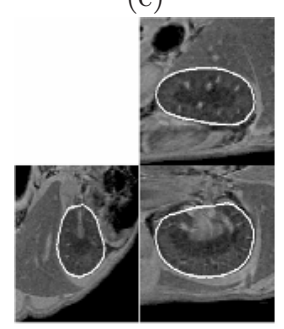

(f)

Fig. 3. Two examples of kidney segmentation. (a,d) Initial model, (b,e) final Model, and $(c, f) 2 \mathrm{D}$ projections of final model[D].

\subsection{Linear and Nonlinear Registration}

Image registration provides spatial normalization of the data by warping one volume onto another allowing for organ comparison. In voxel based image registration we seek the spatial transformation $t$ that maximizes the similarity between the images

$$
\max _{t} s\left(I_{1}, t\left(I_{2}\right)\right)
$$




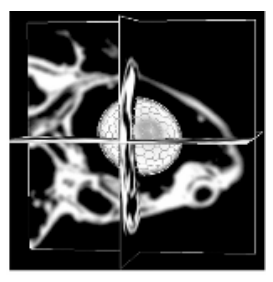

(a)
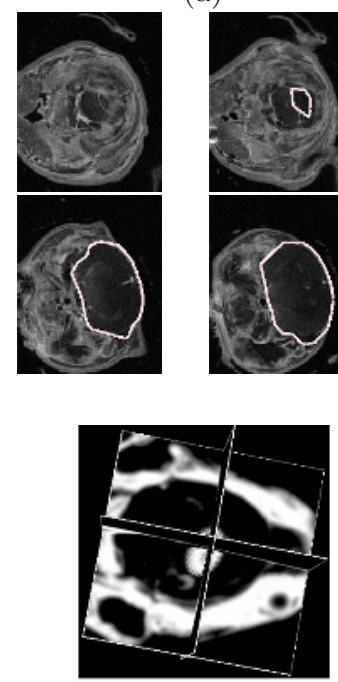

(e)

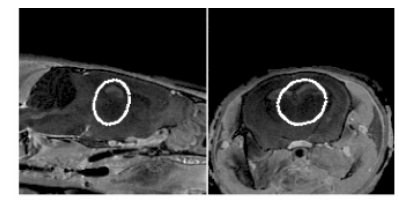

(h)

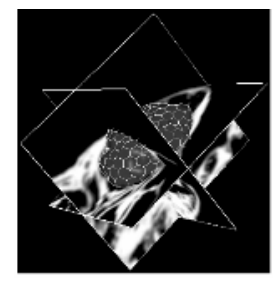

(b)
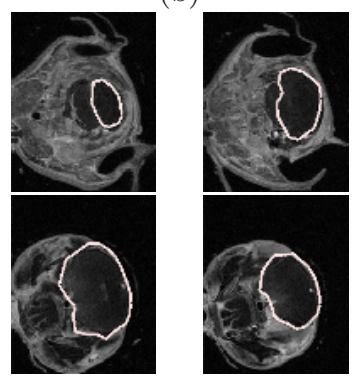

(d)

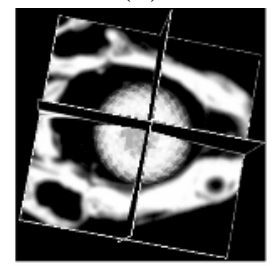

(f)

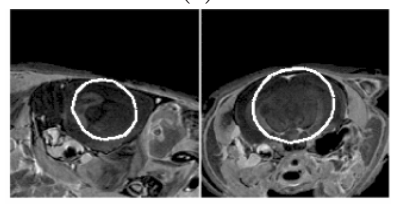

(i)

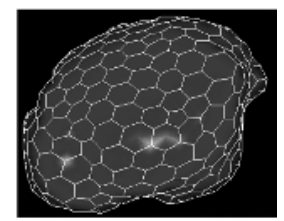

(c)
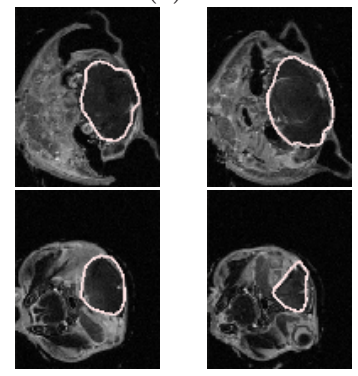

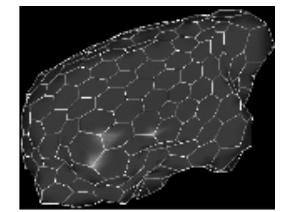

(g)

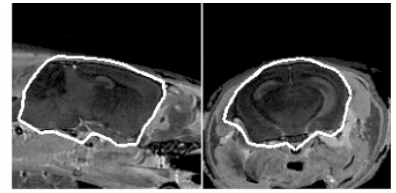

(j)

Fig. 4. Brain segmentation examples (a,e) Inital model, (b,f) 3D deformation, (c,g) final model, (d) 2D projections of final segmentation result at selected slices, and (h-j) $2 \mathrm{D}$ projections showing progress of segmentation[D].

where $s$ is a similarity function, $I_{1}$ and $I_{2}$ are the target and source images, respectively. We adopt a multi-resolution approach by linearly registering the images (Figure 5) then performing successive nonlinear registration steps on an image pyramid (Figure 6a-c). We express the spatial deformations using a displacement field that describes where each point in the source volume moves to in the target volume (Figure 6d-f). Simplex optimization of cross-correlation similarity was used. 


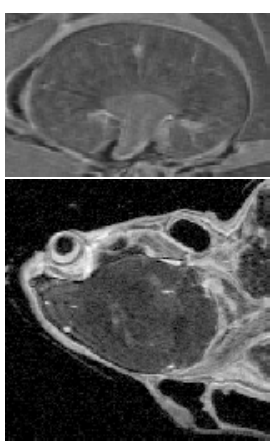

(a)

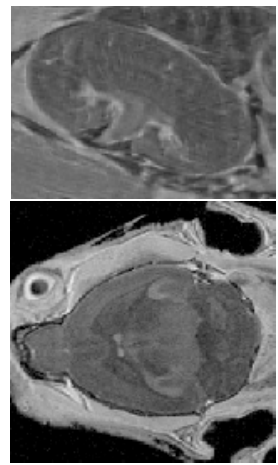

(b)

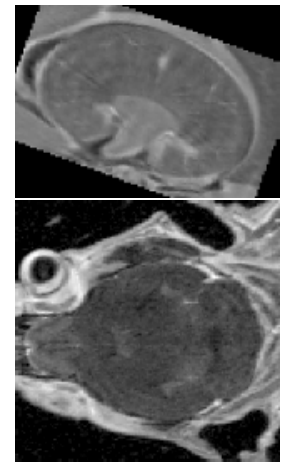

(c)

Fig. 5. Linear registration of a pair of mouse kidneys (top) and brains (bottom), (a) source (b) target, (c) source linearly registered to target[D]

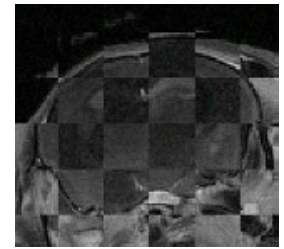

(a)

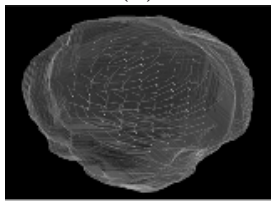

(d)

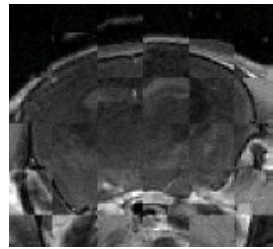

(b)

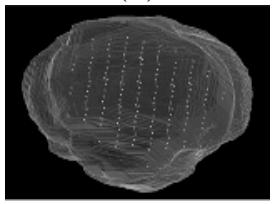

(e)

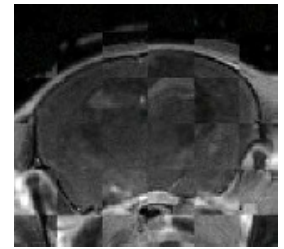

(c)

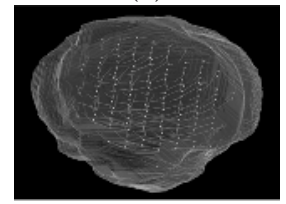

(f)

Fig. 6. Brain registration. Checkerboard comparison of target brain and (a) source, (b) linearly registered, and (c) nonlinearly registered source brain images. (d) Segmented surface before and after registration with linear, (e) nonlinear, (f) and both displacement fields superimposed.

\subsection{Volume Measurement}

In addition to measuring the total volume of segmented structures, we characterize the 3D local volume changes between the same organ in different mice. The local volume changes are calculated at points inside the organ of interest, as found by segmentation, from the displacement field obtained by nonlinear registration. The deformation field encodes how each tri-ordinate $(u, v, w)$ vertex of a $3 \mathrm{D}$ uniform lattice in the source image space is displaced to $(U, V, W)$ in the target. The local volume change values $d(u, v, w)$ are calculated according to

$$
d(u, v, w)=\operatorname{det}\{\nabla[U(u, v, w), V(u, v, w), W(u, v, w)]\}
$$


where $\nabla$ denotes the gradient of a vector function and $\operatorname{det}($.$) denotes the determi-$ nant of a matrix [3]. The deformation function $d(u, v, w)$ measures the expansion or shrinkage at $(u, v, w)$ required to register the structures following linear alignment.

By performing the local volume change measurements on the corpus callosum (CC) of mice heterozygous and homozygous for a null allele of the receptor tyrosine kinase EphB2, we were able to visualize the relative volume reduction (Figure $7(\mathrm{a}-\mathrm{c}))$. The figure clearly shows CC shrinkage in the mid-sagittal region (darker areas) in EphB2 null mice. This is also visible in the coronal MR cross sections (Figure $7(\mathrm{~d}, \mathrm{e})$ ), where the CC shrinks (almost disappears) towards the mid-sagittal region in the knockout mouse[5].

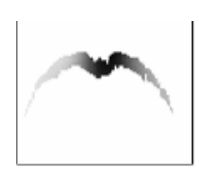

(a)

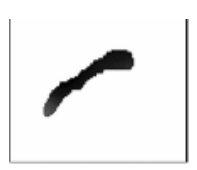

(b)

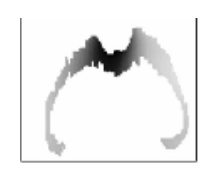

(c)

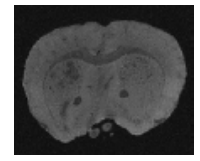

(d)

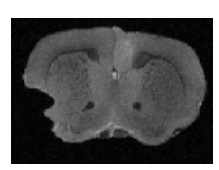

(e)

Fig. 7. Local volume change measurements shown in (a) coronal, (b) mid-sagittal, and (c) transversal cross sections through wildtype CC. Darker areas mean more volume reduction. MRI coronal cross sections through the (d) EphB2 (+/-) and (e) EphB2 (-/-) murine brains[M2].

\section{Implementation and Hardware and Software Details}

Parallel code for MR reconstruction ran on 192-processor, 178GB RAM, SGI Origin 3800 system. The automated image registration (AIR 5.2.5) toolkit, UCLA, and MINC tools from the Montreal Neurological Institute, McGill University, were used for $3 \mathrm{D}$ diffusion filtering and image registration. The YAV ++ software from INRIA, France, was used for segmentation. Code for calculating local volume change measurements was written in MATLAB, MathWorks. The Amira Software, TGS, was utilized for 3D visualization. Higher level TCL and python scripts were used for gluing the different components.

\section{Conclusion}

We described our MR image analysis pipeline and presented the results obtained so far on mouse kidneys and brains. Our current work is by no means complete; image processing and analysis parameters and schedule (pre-processing, segmentation, registration) need to be fine tuned once mouse perfusion and parallel imaging protocols reach a steady state. Validation on a larger number of image samples is required. Running MR images of a number of normal, inbred mice through the pipeline allows statistical studies. The aim of these studies 
will be to capture the variations of different measurements (total volume, local volume changes, deformation fields, and shape parameters) of normal mice and subsequently detect abnormalities in genetically altered mice.

Acknowledgments. We are grateful to Dr. G. A. Johnson of the Duke Center for In Vivo Microscopy, an NIH/NCRR National Resource (P41 05959), for supplying the 3D whole body image data from the Visible Mouse Project. We also thank Herve Delingette and Jean-Didier Lemarechal of INRIA, France, for sending and helping with the YAV software.

\section{References}

1. Boguski, M. S.: Comparative genomics: the mouse that roared. Nature 420 (2002) 515-516.

2. Christensen, G., Wang, Y., and Chien, K. R.: Physiological assessment of complex cardiac phenotypes in genetically engineered mice. Am. J. Physiol. 272 (1997) 2513-2524.

3. Davatzikos, C. A., Vaillant, M., Resnick, S., Prince, J. L., Letovsky, S., Bryan, R. N.: A Computerized approach for morphological analysis of the corpus callosum. Journal of Computer Assisted Tomography 20(1) (1996) 88-97.

4. Delingette, H.: General object reconstruction based on simplex meshes. International Journal of Computer Vision 32(2) (1999) 111-146.

5. Hamarneh, G., Chen, J., Lifshitz, N., Henderson, J., Henkelman, M.: Local volume changes of the corpus callosum from 3D MR images of wildtype and knockout mouse brains. Int. Soc. Mag. Res. Med., 2003.

6. Johnson, G. A., Cofer, G. P., Gewalt, S. L., Hedlund, L. W.: Morphologic phenotyping with magnetic resonance microscopy: the visible mouse. Radiology 222(3), (2002) 789-793.

7. Kent, G., Iles, R., Bear, C. E., Huan, L. J., Griesenbach, U., McKerlie, C., Frndova, H., Ackerley, C., Gosselin, D., Radzioch, D., O’Brodovich, H., Tsui, L. C., Buchwald, M., and Tanswell, A. K.: Lung disease in mice with cystic fibrosis. J. Clin. Invest 100 (1997) 3060-3069.

8. Maintz, J., Viergever, M.: A Survey of Medical Image Registration. Medical Image Analysis 2(1) (1998) 1-37.

9. McInerney, T., Terzopoulos, D.: Deformable Models in Medical Image Analysis: A Survey. Medical Image Analysis 1(2) (1996) 91-108.

10. Montagnat, J., Delingette. H.: Globally constrained deformable models for 3D object reconstruction. Signal Processing 71(2) (1998) 173-186.

11. Perona P., Malik J.: Scale-Space and Edge Detection Using Anisotropic Diffusion. IEEE Trans. PAMI 12(7) (1990) 629-639.

12. Russell, L. B., Bangham, J. W., Stelzner, K. F., and Hunsicker, P. R.: High frequency of mosaic mutants produced by N-ethyl-N-nitrosourea exposure of mouse zygotes. Proc. Natl Acad. Sci. USA 85 (1988) 9167-9170.

13. Waterston, R. H., et. al. (100+ authors): Initial sequencing and comparative analysis of the mouse genome. Nature 420 (2002) 520-562.

14. Yang, M., Charlton, B., Gautam, A. M.: Development of insulitis and diabetes in B cell-deficient NOD mice. J. Autoimmun. 10 (1997) 257-260. 